\title{
INVESTIGATION OF GAS GAIN OF GEM-FOIL USED IN LOW ENERGY RADIOACTIVE BEAM EXPERIMENT
}

\author{
NGUYEN NGOC DUY \\ Dong Nai University \\ LE HONG KHIEM \\ Institute Of Physics, VAST
}

\begin{abstract}
Nuclear reaction measurement with radioactive beam at low energy plays an important role in nuclear astrophysics and nuclear structure. The trajectory of particle beams can be obtained by using an active gas target, multiple-sampling and tracking proportional chamber (MSTPC), as a proportional counter. Because of intensity of low energy radioactive beam, in the stellar reaction such as $(\alpha, p),(p, \alpha), \ldots$ it is necessary to increase the gain for the counter. In this case, a gas electrons multiplier (GEM) foil will be used, so the proportional counter is called GEM-MSTPC. The efficient gas gain of GEM foils which relates to foil thickness and operating pressure was investigated with two type of the foils, 400 um and 200 um, in argon (70\%) - carbon dioxide (30\%) mixture.
\end{abstract}

\section{INTRODUCTION}

An active gas detector, multiple-sampling and tracking proportional chamber (MSTPC) [1] has advantage for direct measurement of stellar reaction using radioactive ion (RI) beams. The trajectory of particles in three-dimension and energy of particles can be measured with high accuracy. However, it is necessary to get high gain for MSTPC since the RI beam has low intensity. One or more GEM foils [2] are mounted between the drift space and the readout pads to increase the output signal. GEM foils works as an amplifier to amplify the ionization electrons in the drift region of MSTPC so it is very important to know the gain of GEM foils.

The gas gain of GEM foil as a function of high voltage (corresponding to operating electrict field) applied for GEM. Here, the operating electric field $E_{G E M}$ is defined as:

$$
E_{G E M}=\frac{\Delta V}{d \cdot p}
$$

where $\Delta V$ is high voltage applied for GEM-up and GEM-down, $d$ is the thickness of GEM foil and $p$ is the operating gas pressure.

In this paper, we present the gas gain of two type of GEM-foil with different thickness, $400 \mathrm{um}$ and $200 \mathrm{um}$, and operating pressure. 


\section{EXPERIMENT SETUP}

The experiment was performed at Center for Nuclear Study (CNS), the University of Tokyo. Gas gain of GEM-foils was obtained and the foils were installed in the GEMMSTPC for direct measurement of stellar reaction ${ }^{30} \mathrm{~S}(\alpha, \mathrm{p})$ at CNS Radioactive Ion Beam (CRIB) separator [3]. Because of high Coulomb barrier, the $\mathrm{Ar}-\mathrm{CO}_{2}$ (30\%) mixture gas was used for background run in the ${ }^{30} \mathrm{~S}(\alpha, \mathrm{p})$ experiment.

Two types of Japanese GEM foil with $400 \mathrm{um}$ and $200 \mathrm{um}$ in thickness [4] and 110 $\mathrm{mm} \times 190 \mathrm{~mm}$ in dimension were used in this experiment. The structure of active target chamber includes a top plate, the GEM foil and pad readout, figure 1. The GEM foils have two sides, GEM-up and GEM-down, they were separated by an insulator $400 \mathrm{um}$ and $200 \mathrm{um}$, respectively. The drift region between top plate and GEM-up was $1 \mathrm{~cm}$ and the space from GEM-down to pad readout was $2 \mathrm{~mm}$. The GEM-down was applied a limited voltage at $135 \mathrm{~V}$ with $0 \mathrm{~V}$ at pad readout. The signal from pad readout was connected to IH 142 Ortec preamplifier, which has conversion gain $1 \mathrm{~V} / \mathrm{pC}$. An $\alpha$-source ${ }^{241} \mathrm{Am}$ which have energy $5.5 \mathrm{MeV}$ and injection rate $10^{5}$ pps were used. We performed two runs for the measurement of gas gain in $\mathrm{Ar}-\mathrm{CO}_{2}(30 \%)$ mixture gas, one for 400um-GEM foil and 200um-GEM foil at the same electric field condition and another for 200um-GEM foil at different pressure.

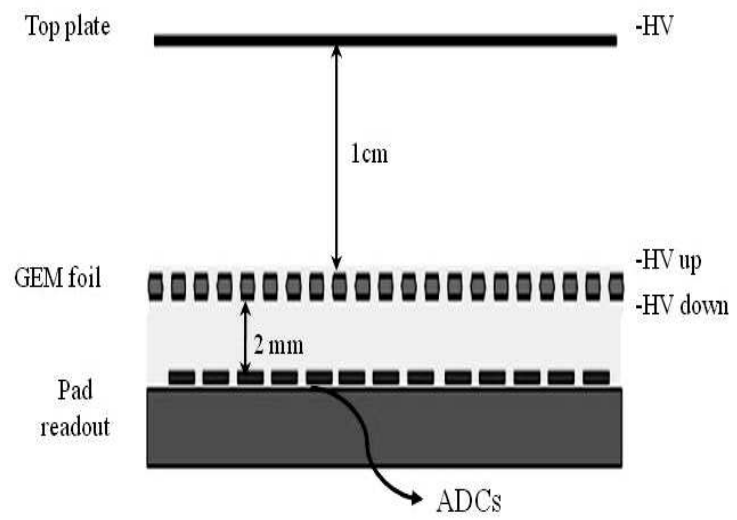

Fig. 1. View of the experiment for testing gas gain of $200 \mathrm{um}-$ and $400 \mathrm{um}-\mathrm{GEM}$ foils in $\mathrm{ArCO}_{2}$

\section{RESULTS}

The measured gas gain of 400um- and 200um- GEM foils was showed in Fig. 2. At the same operating electric field, the value of thicker foil is larger than other one because of the large avalanche region. This is also consistent with previous works reported in ref. [5]. For the $400 \mathrm{um}$ foil, the gas gain is one order higher than $200 \mathrm{um}$ foil. Therefore, it is suitable to use the thicker foil in the experiment which requires a high gain. For example, with the gain need to be 278 for measuring ${ }^{30} \mathrm{~S}$ particles beam in the ${ }^{30} \mathrm{~S}(\alpha, \mathrm{p})$ reaction experiment, we can use a $400 \mathrm{um}$ single foil with the electric field around $130 \mathrm{kV} / \mathrm{cm} / \mathrm{atm}$. 
According to the measured results, the gas gain does not decrease even when the electric field in drift region is very high.
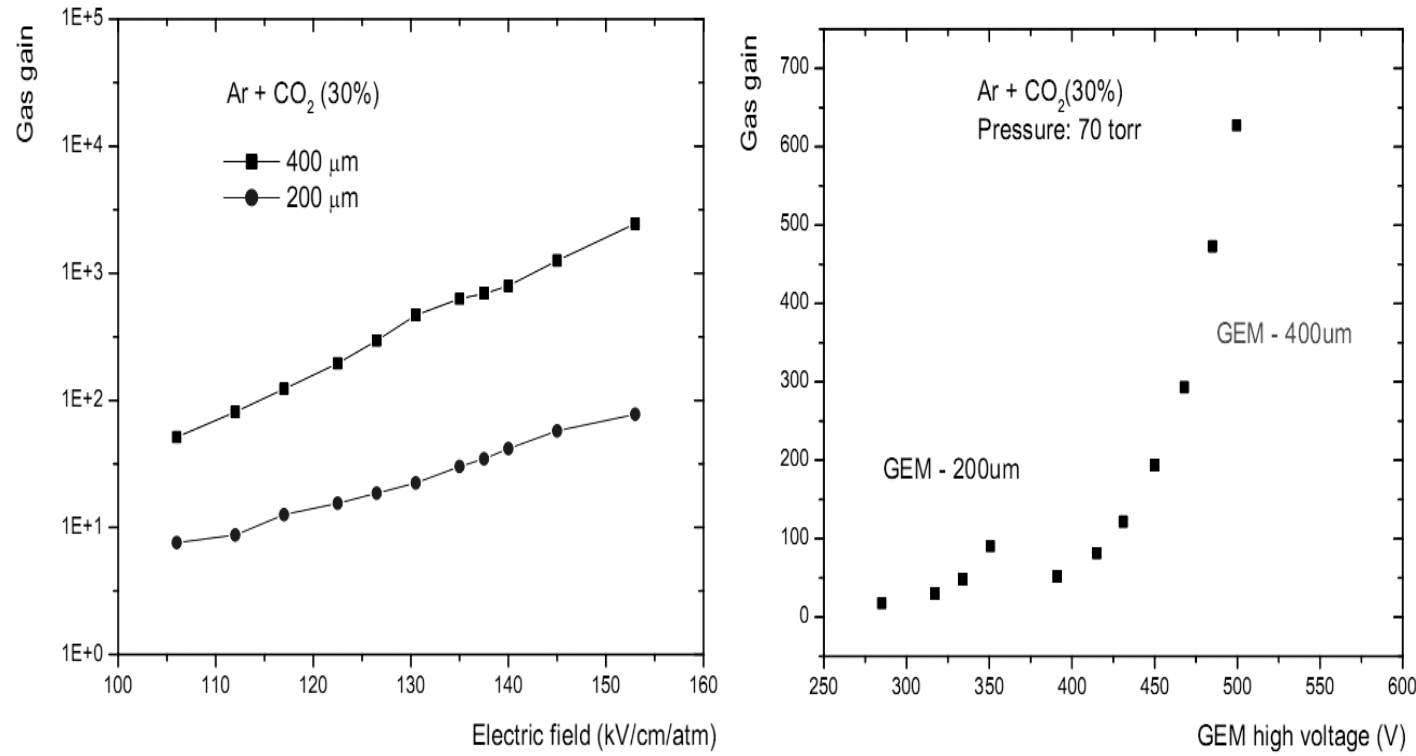

Fig. 2. The gas gain as a function of operating electronic field between two sides of the GEM

Fig. 3. The gas gains as a function of voltage between two sides of GEM foils. foil.

The high voltage applied for GEM foils was also investigated, the result is shown in Fig. 3. At the same pressure 70 torr, the voltage applied for thicker GEM is higher and the gas gain can be obtained larger because of the large avalanche region between two sides of GEM foil. However, there was a threshold for applied voltage of the GEM foils. The gas gain increases proportionally to high voltage but discharge occurs in GEM foils when the voltage reaches over the threshold, at $520 \mathrm{~V}$ for $400 \mathrm{um}$-GEM and $360 \mathrm{~V}$ for 200 um-GEM. We could not get higher gain with higher voltage which was over those thresholds. In addition, the output signal just could be observed when the voltage was applied more than a critical value. The value was different for two types of the foils we carried out and the critical voltage which was required for the thicker foil was also higher than that for the thinner. They were $360 \mathrm{~V}$ and $250 \mathrm{~V}$, respectively.

Gas operating pressure is also play an important role in gas gain of the GEM foil. In the experiment, with the $200 \mathrm{um}$ GEM foil, we changed gas pressure and measured the values. In the work, the gain of the GEM foil as a function of operating pressure was investigated. The result on figure 4 was obtained in the measurement at $410 \mathrm{~V}$ and at the same thickness $200 \mathrm{um}$. It indicates that if the needed gain is low, we can use higher pressure. And thus, it is possible to use high pressure to measure the trajectory of heavy particle, whose deposit energy in the axis is large enough for the resolution.

Because of operating electric field plays an important role for transmission of drift electrons produced by ionization, its effect on gas gain need to be considered. The Fig. 5 

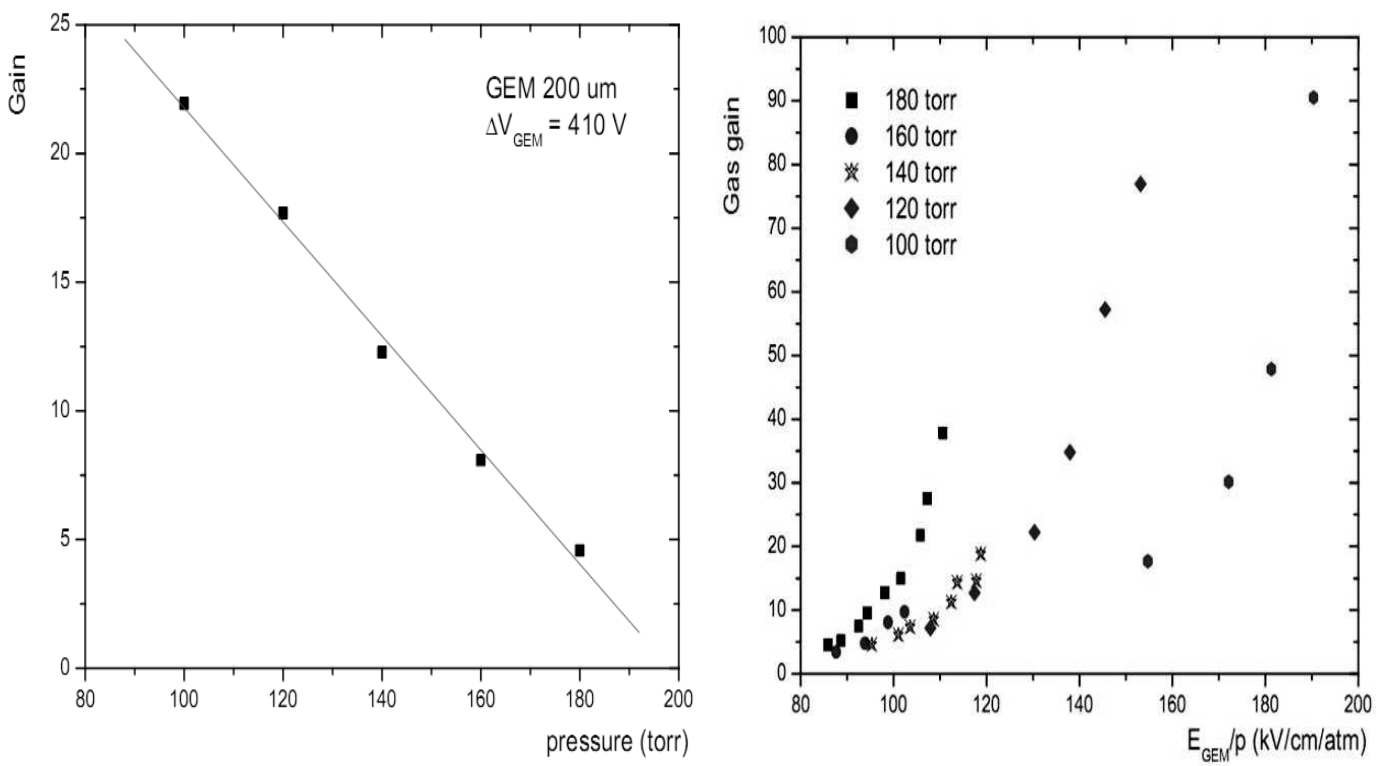

Fig. 4. The gain of GEM foil $200 u m$ at $410 \mathrm{~V}$ with different pressures.

Fig. 5. The gas gain as a function of operating electric field with different operating pressure.

shows the correlation of gain and the operating electric field. The results indicate that, at the same operating electric field, the higher operating pressure is, the larger gas gain could be obtained. The ionization of particles in a high pressure gas should be easy to provide a lot of electrons in the avalanche region. However, at low operating pressure, the maximum value of gas gain will be obtained very high but we have to apply much more voltage. It is difficult to get a strong electric field without discharge. Because of those reasons, some GEM foils will be mounted (multi-GEM foils) [6] so that the gas gain of GEMs can be enough to measure particles, specially, the light particles whose energy loss is a few of $\mathrm{keV}$.

\section{SUMMARY}

We measured gas gains with single Japanese GEMs, $200 \mathrm{um}$ and $400 \mathrm{um}$, in different pressure Ar+CO2 (30\%) gas. While the gas gain 200 um-GEM was less than $10^{2}$, the gain of $400 \mathrm{um}-\mathrm{GEM}$ can be higher than $10^{3}$. The gas gain of GEM foils depends on the thickness of foils, electric field and operating pressure. The thicker GEM foils can be applied higher electric field so the maximum-gain should be higher than the thinner one. If the operating pressure is lower, the maximum-gain can be obtained with higher value when the electric field increases. As the results, we can use the 400um-GEM foils to measure light particles which need a gain approximately $10^{3}$ and other for heavy particles. In order to get larger range of gas gain, the low pressure is a candidate for measurement. Some experiments need a higher gas gain to get clearly output signals, it is necessary to use a multi-GEM foils. The value of multi-GEM foils of these types of GEM foils will be estimated and compared with other works. 


\section{ACKNOWLEDGMENT}

We would like to express our gratitude to Prof. Kubono and all CRIB-collaborators of the University of Tokyo, who gave us the possibility to perform the experiment. We would like to thank operators of AVF cyclotron at RIKEN and engineers of CRIB for the beams used in the experiment. The financial support from the University of Tokyo is gratefully acknowledged. This work was partial financial sponsored by the Vietnams National Foundation for Science and Technology Development (NAFOSTED) under Contract No. 103.04.54.09.

\section{REFERENCES}

[1] T. Hashimoto et al., Nucl. Instr. and Meth. A556 (2006) 339-349.

[2] F. Sauli, Nucl. Instr. and Meth. A386 (1997) 531.

[3] S. Kubono, Nucl. Phys. A693 (2001) 221.

[4] Scienergy Co. Ltd. (info@scienergy.jp). ¡hhttp://www.scienergy.jpi

[5] R. Chechik, A. Breskin, C. Shalem, Nucl. Instr. and Meth. A533 (2005) 35.

[6] A. Buzulutskov, Nucl. Instr. and Meth. A494 (2002) 148-155.

Received 01 June 2011. 